\title{
Mapeamento Sistemático na Literatura Acadêmico-Científica sobre Abordagens para Formação de Grupos em E-Learning
}

Title: Systematic Mapping of the academic and scientific literature on Approaches for Groups Formation in E-Learning

Felipe Oliveira Miranda Cunha

Programa de Pós-Graduação em Informática

Universidade Federal da Paraíba (UFPB)

João Pessoa - PB, Brasil

felipeoliveira.ufpb@gmail.com

\author{
Clauirton de Albuquerque Siebra \\ Programa de Pós-Graduação em Informática \\ Universidade Federal da Paraíba (UFPB) \\ João Pessoa - PB, Brasil \\ clauirton@ci.ufpb.br
}

\begin{abstract}
Resumo Este trabalho apresenta os resultados de um mapeamento sistemático realizado a partir de artigos cientificos que versam sobre a formação de grupos de estudantes que participam de processos de aprendizagem colaborativa. O objetivo é investigar as pesquisas que relacionam abordagens e estratégias de formação de grupos no contexto do ensino a distância. Esta pesquisa mostra que as publicações estão focadas na análise do contexto das interações de estudantes em colaboração e no aprimoramento de algoritmos para otimização de grupos. Especificou-se tipos de grupos para posterior experimento em agrupamento homogêneo, agrupamento heterogêneo e complementar por habilidades em programação a partir da modelagem matemática e implementação de algoritmo de agrupamento. O resultado deste trabalho produz fundamentação teórica para a investigação de novas abordagens para formação de grupos a partir de perfis de estudantes criados sob critérios e preferências ainda não explorados, assim como uma análise mais precisa das interações e o impacto da colaboração no desempenho de estudantes em ambientes de educação a distância.
\end{abstract}

Palavras-Chave: Formação de grupos, E-Learning, Mapeamento sistemático.

Keywords: Group formation, E-Learning, Systematic Mapping. 


\section{Introdução}

Pesquisas têm investigado o potencial da Aprendizagem Colaborativa Apoiada por Computador, do inglês Computer Supported Collaborative Learning - CSCL, como instrumento de aumento do poder cognitivo e da produtividade na universidade [1,6]. A CSCL preocupase em como os estudantes utilizam sistemas computacionais para trabalhar em grupo e como se organizam conjuntamente para o estudo colaborativo, através de modelos de ensino e de aprendizagem E-learning.

Grupos são coleções de indivíduos que interagem entre si, compartilhando interesses comuns e representando funções específicas. Eles podem ser criados de diferentes formas e possuir tamanho, duração e objetivos distintos. No trabalho em grupo, um problema complexo é dividido em subproblemas e os membros trabalham juntos, visando encontrar resultados parciais [3], negociando e compartilhando entendimentos relevantes à resolução de um problema [11].

Pesquisadores enfatizam a necessidade de que os estudantes partam do aprendizado individual para o estudo em grupo [5]. Trabalhar colaborativamente para resolver problemas pode ter inúmeras vantagens. Através do ensino recíproco $[9,12]$ a troca de conhecimento é estabelecida mais fortemente e alunos mais experientes podem apoiar os demais. Desse modo, acredita-se no trabalho em grupo para efetivamente potencializar o aprendizado compartilhado e elevar a qualidade do processo educativo em E-learning.

A abordagem utilizada para a formação de grupos é um aspecto significativo para o sucesso do trabalho colaborativo a distância. Estabelecer critérios e preferências constitui um passo importante para a formação de grupos. Esses critérios são considerados para determinar as características de um grupo, sendo que alunos podem ser agrupados por diferentes dimensões. Algumas abordagens afirmam que essa tarefa deva ser compartilhada entre o professor e os alunos [7]. Outras reforçam a ideia de que a formação auto selecionada pode ter melhores resultados que a aleatória [2]. A proposta de [4], por exemplo, considera critérios gerais e específicos essenciais para a atribuição dos membros. Entretanto, garantir que a maioria das preferências seja atendida não é uma tarefa pedagogicamente fácil, pois as características e as necessidades de cada turma podem variar.

Este trabalho objetiva investigar de forma sistemática os estudos que relacionam abordagens e estratégias de formação de grupos em E-learning para fornecer dados que embasem o desenvolvimento de estudos mais específicos sobre aprendizagem colaborativa suportada por computador. Utilizou-se as etapas de mapeamento sistemático da literatura de acordo com [10]. O estudo pretende responder ao seguinte questionamento: em que medida as publicações na área têm contribuído com abordagens e estratégias, dentro do contexto internacional, em formação de grupos para a aprendizagem colaborativa?

A seção 2 contextualiza a formação de grupos na educação a distância. A seção 3 descreve a metodologia do estudo. A seção 4 apresenta os resultados obtidos, bem como a análise e discussão. Finalmente, a seção 5 apresenta as conclusões.

\section{Formação de grupos na educação a distância}

A Educação a Distância (EaD) via Internet consiste na modalidade E-Learning de ensino e de aprendizagem através da comunicação mediada por tecnologias computacionais, com estudantes e professores desenvolvendo atividades educativas em lugares e tempos distintos [9], através de ambientes virtuais de aprendizagem. Esses ambientes são concebidos como espaços de gerenciamento de cursos e de interação entre os usuários. Configuramse como as salas de aulas virtuais e integram recursos multimidiáticos para estimular maior participação e comunicação entre os envolvidos. Uma das preocupações da $\mathrm{EaD}$ é garantir o pleno desenvolvimento da aprendizagem, pois demanda uma postura do aluno, agora virtual, pautada na cooperação, autonomia, capacidade para o trabalho em equipe e aprendizado colaborativo.

Pensar estratégias de formação de grupos para apoiar a aprendizagem colaborativa na $\mathrm{EaD}$ pressupõe considerar as possibilidades de uso pedagógico dos ambientes virtuais [1]. Ferramentas de comunicação online, como o fórum, chat, quiz, envio de arquivos, entre outros recursos síncronos e assíncronos, podem potencializar o ensino e a aprendizagem colaborativa, pois suportam diferentes formas de interação, permitindo a efetiva participação e desenvolvimento do aluno para competências, habilidades, atitudes e hábitos necessários à sua formação. Entretanto, para o uso eficaz e eficiente desses recursos no apoio ao estudo colaborativo é preciso definir uma abordagem de formação que atenda aos objetivos de aprendizagem pretendidos.

Tomando como perspectiva o papel do tutor-mediador na maioria dos programas de Educação a Distância no Brasil [14], o conhecimento do tutor e suas preferências em relação à formação de grupos pode ser um caminho para atribuir estudantes em grupo de forma coerente, tendo em vista a sua intensa relação com o potencial, as dificuldades e as necessidades de cada aluno. Outras 
abordagens para apoiar o processo de formação de grupos na EaD utilizam o perfil e o contexto de alunos nos ambientes virtuais de aprendizagem, como visto em [8]. Diante disso, o presente estudo, além de possibilitar conhecer da literatura as diferentes abordagens de formação de grupos, permitirá o conhecimento dessa estratégia de aprendizagem aplicada ao contexto de ensino a distância.

\section{Metodologia}

A construção de uma base de conhecimento científico sobre determinado tema é, em geral, fundamentada na análise de estudos anteriores. O Mapeamento Sistemático da Literatura - MSL -, a partir de alguns estudos relevantes, utilizam, na maioria dos casos, a análise qualitativa das narrativas obtidas das leituras, de modo a contribuir para o aprofundamento de um campo científico existente ou analisar metodologicamente os resultados de estudos realizados [13].

O mapeamento sistemático é um método que consiste na construção de um esquema ou estrutura de classificação de um campo de estudo [10]. Esta seção descreve o processo de mapeamento, utilizando-se do processo metodológico proposto em [10], para fornecer uma visão mais ampla de pesquisa acerca do tema formação de grupos de aprendizagem e as abordagens utilizadas para a atribuição de estudantes em grupo.

\subsection{Estratégia de busca}

O primeiro passo para utilizar a MSL consiste na definição dos parâmetros para o processo de mapeamento sistemático da literatura de formação de grupos de aprendizagem. Como estratégia de busca, a partir do tema e da questão central que motiva o estudo, deve-se definir os conceitos-chave, as Strings de busca, os portais de busca e os critérios de exclusão. O processo de busca utilizado neste trabalho será o automático, definindo-se, para isso, as Strings que representam o conjunto de termos identificados a partir da questão de pesquisa, visando a obtenção de uma maior cobertura da literatura existente.

\subsection{Definição dos conceitos-chave}

Para a construção do conjunto de termos utilizados na busca automática, os conceitos-chave devem ser definidos. Esses conceitos podem ser identificados a partir da questão motivadora do estudo, compreendido por: "em que medida as publicações na área têm contribuído com abordagens ou estratégias, dentro do contexto internacional, em formação de grupos para a aprendizagem colaborativa?". Para identificar os conceitos, parte-se da análise dos construtos centrais da questão, a saber: "abordagens ou estratégias" e "formação de grupos para a aprendizagem colaborativa". De posse desses construtos, pode-se destacar os seguintes conceitos-chave:

- Abordagem em grupo

- Estratégia em grupo

- Formação de grupos

- Aprendizagem em grupo

- Estudantes em grupo

- Aprendizagem colaborativa

- Atribuição de grupos

- Formação de grupos de aprendizagem

\subsection{Termos utilizados na busca (string)}

A String de busca consiste na combinação dos conceitos-chave que, neste trabalho, foi utilizada apenas na língua inglesa, utilizando-se os operadores lógicos "OR", que representa uma disjunção, e " $A N D$ ", representando uma conjunção. A String utilizada compreendeu : "group approach" OR "group strategy" OR "group formation" AND "group learning" OR "group students" OR "collaborative learning" OR "learning groups".

\subsection{Portais de busca}

Para as buscas automáticas neste estudo os engenhos foram definidos conforme relevância na área da Ciência da Computação, a saber: ACM Digital Library (http://dl.acm.org/); IEEE Xplore (http://ieeexplore.ieee.org/); $\quad$ Scopus (http://www.scopus.com/); $\quad$ ScienceDirect (http://www.sciencedirect.com/).

\subsection{Critérios de inclusão e exclusão}

Os critérios de exclusão compreendem: artigos convidados, tutoriais, relatórios de workshop, teses, dissertações, relatórios técnicos, livros; documentos que não sejam artigos completos; artigos duplicados, que já foram encontrados em outras fontes; trabalhos sem os arquivos encontrados; estudos secundários, e; estudos que não correspondem à questão da pesquisa.

Como critérios de inclusão, pretende-se incluir os artigos científicos completos que tenham foco em abordagens e ou estratégias para formação de grupos de aprendizagem, incluindo os que apresentam resultados de experimentos e sua análise.

\section{Resultados}

Nesta seção são apresentados os resultados encontrados no processo de mapeamento sistemático realizado e análise dos trabalhos selecionados que apresentam abor- 
dagens para formação de grupos colaborativos.

\subsection{Informações gerais dos trabalhos primá- rios}

O processo de busca retornou um total de 86 (oitenta e seis) trabalhos, extraindo-se desses os dados gerais por portal de busca: título, autores, abordagem de pesquisa, local de pesquisa, ano de publicação, país de origem dos pesquisadores e portal. O processo de obtenção dos trabalhos consistiu na filtragem dos trabalhos potencialmente relevantes. Após essa etapa, realizou-se análise dos estudos incluídos, considerados relevantes do ponto de vista da contribuição da pesquisa com a questão norteadora do presente estudo, compreendendo um total de 29 (vinte e nove) artigos científicos. Em seguida, realizou-se a leitura completa do conteúdo dos trabalhos, buscando identificar as informações específicas de cada trabalho, conforme descrito na seção 4.2 .

A Tabela 1 apresenta resumidamente a evolução do processo de obtenção das publicações incluídas no estudo. Na coluna da esquerda estão dispostos os portais de busca utilizados. A coluna seguinte apresenta a quantidade de artigos retornados na primeira etapa do mapeamento sistemático, por meio das strings de busca. Nas demais colunas estão dispostos os resultados da primeira seleção, com os trabalhos potencialmente relevantes, e da segunda seleção, com os quantitativos de trabalhos excluídos, compreendidos nos trabalhos duplicados, irrelevantes e indisponíveis, bem como os trabalhos primários, considerados por apresentar contribuições originais para área.

\begin{tabular}{|c|c|c|c|c|c|c|}
\hline \multicolumn{7}{|c|}{ Seleção dos Trabalhos Primários } \\
\hline \multirow[t]{3}{*}{ Portais } & \multirow{3}{*}{$\begin{array}{l}\text { Trabalhos retor- } \\
\text { nados }\end{array}$} & $1^{\text {a }}$ Seleção & $2^{\mathrm{a}} \mathrm{Se}$ & & & \\
\hline & & \multirow{2}{*}{$\begin{array}{l}\text { Potencialmente } \\
\text { relevantes }\end{array}$} & \multicolumn{3}{|c|}{ Excluídos } & \multirow{2}{*}{$\begin{array}{l}\text { Incluídos } \\
\text { Trabalhos primá- } \\
\text { rios }\end{array}$} \\
\hline & & & 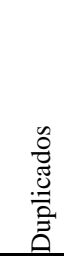 & 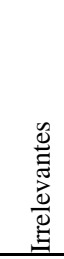 & 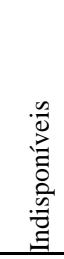 & \\
\hline ACM Digital Library & 13 & 10 & - & 3 & - & 7 \\
\hline IEEE Xplorer & 34 & 19 & 1 & 4 & - & 14 \\
\hline Scopus & 28 & 20 & 9 & 1 & 6 & 4 \\
\hline Science Direct & 11 & 9 & - & 5 & - & 4 \\
\hline Total & 86 & 58 & 10 & 13 & 6 & 29 \\
\hline
\end{tabular}

Tabela 1: Evolução do processo de seleção dos trabalhos primários

Verificou-se que, dos trabalhos retornados, o portal IEEE Xplorer foi o que apresentou número mais expressivo, compreendido em 34 (trinta e quatro) artigos, seguido do portal Scopus com 28 (vinte e oito) trabalhos, o portal ACM Digital Library com 13 (treze) e, com menor quantidade, o Science Direct, compreendido em 11(onze) artigos. Em relação ao quantitativo de trabalhos potencialmente relevantes, o portal IEEE Xplorer foi reduzido para 19 (dezenove), o portal Scopus para 20 (vinte), o portal ACM para 10 (dez) e o Science Direct para 9 (nove).

Para a primeira seleção, compreendida pela obtenção dos trabalhos relevantes, realizou-se leitura e análise dos títulos e resumos das publicações retornadas. A análise dos trabalhos objetivou identificar os trabalhos que traziam contribuições do ponto de vista de abordagens e estratégias para formação de grupos de aprendizagem colabo- rativa.

Para a segunda seleção, que objetivou identificar os trabalhos excluídos e incluídos, realizou-se leitura e análise do resumo, da introdução e da conclusão dos artigos obtidos na etapa anterior. A partir dessa segunda seleção, verificou-se 9 (nove) trabalhos duplicados no portal Scopus e 1 (um) no IEEE Xplorer, 13 (treze) trabalhos irrelevantes nos portais ACM (3), IEEE Xplorer (4), Scopus (1) e Science Direct (5), e 6 (seis) trabalhos indisponíveis (portal Scopus), todos esses considerados como trabalhos excluídos.

Os trabalhos incluídos, considerados como trabalhos primários, foram 7 (sete) no portal ACM, 14 (quatorze) no portal IEEE Xplorer e 4 (quatro) trabalhos em cada um dos portais Scopus e Science Direct.

A Figura 1 apresenta a distribuição temporal das pu- 
blicações com o quantitativo de trabalhos por ano.

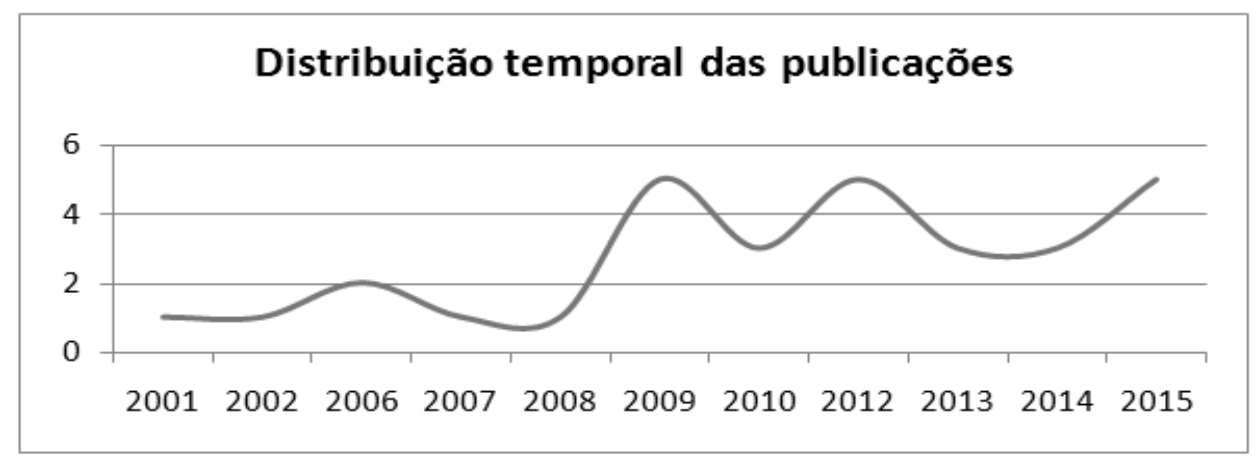

Figura 1: Distribuição temporal dos trabalhos primários

Embora muitos trabalhos retornados tenham sido publicados em diferentes anos, as publicações consideradas como trabalhos primários compreenderam o período de tempo de quinze anos, abrangendo os anos de 2001 a 2015. Verificou-se um número expressivo de publicações entre os anos de 2009 a 2015. Percebe-se, deste modo, que as publicações que apresentam estudos na área são em sua grande maioria recentes.

Com relação aos países de origem dos autores das publicações consideradas como trabalhos primários, verificou-se que, conforme Figura 2, a Espanha lidera a lista com uma frequência de 18 (dezoito) pesquisadores na área, representando $20,7 \%$ do total, seguida de Brasil com 11 (onze) pesquisadores, representando 12,6\%, Japão com 10 (dez) pesquisadores, representando 11,5\%, Inglaterra com 8 (oito), equivalendo a 9,2\%, seguidos dos Estados Unidos e Taiwan com 7(sete) , representando $8 \%$, Alemanha com 6 (seis), representando 7\%, Iran com 5 (cinco) (5,6\%), Austrália com 4 (quatro) (4,6\%), Canadá com 3 (três) (3,4\%), Argentina e Grécia com 2 (dois) $(2,3 \%)$ e Áustria, Colômbia, Eslováquia e Índia com 1 (um)pesquisador, representando $1,1 \%$, dentro das publicações que realmente propõem uma abordagem para formação de grupos colaborativos.

Do ponto de vista de continente, observa-se que há uma grande concentração de pesquisadores no continente europeu, compreendendo $41,3 \%$ do total de pesquisadores das publicações. As pesquisas são bem expressivas também na América e na Ásia, que representam uma concentração de $27,6 \%$ e $26,4 \%$ de pesquisadores interessados, respectivamente. Finalmente, observa-se poucas pesquisas realizadas nos continentes da Oceania com $4,6 \%$ e da África, que não apresentou publicações de acordo com os parâmetros da busca sistemática. A Figura 2 ilustra 16 (dezesseis) países de origem dos pesquisadores e o número de pesquisadores.
$\mathrm{Na}$ sequência foram analisadas as conferências e ou periódicos de origem das publicações. A Figura 3 ilustra o gráfico com a quantidade de publicações por conferência e revista, apresentando-as por siglas.

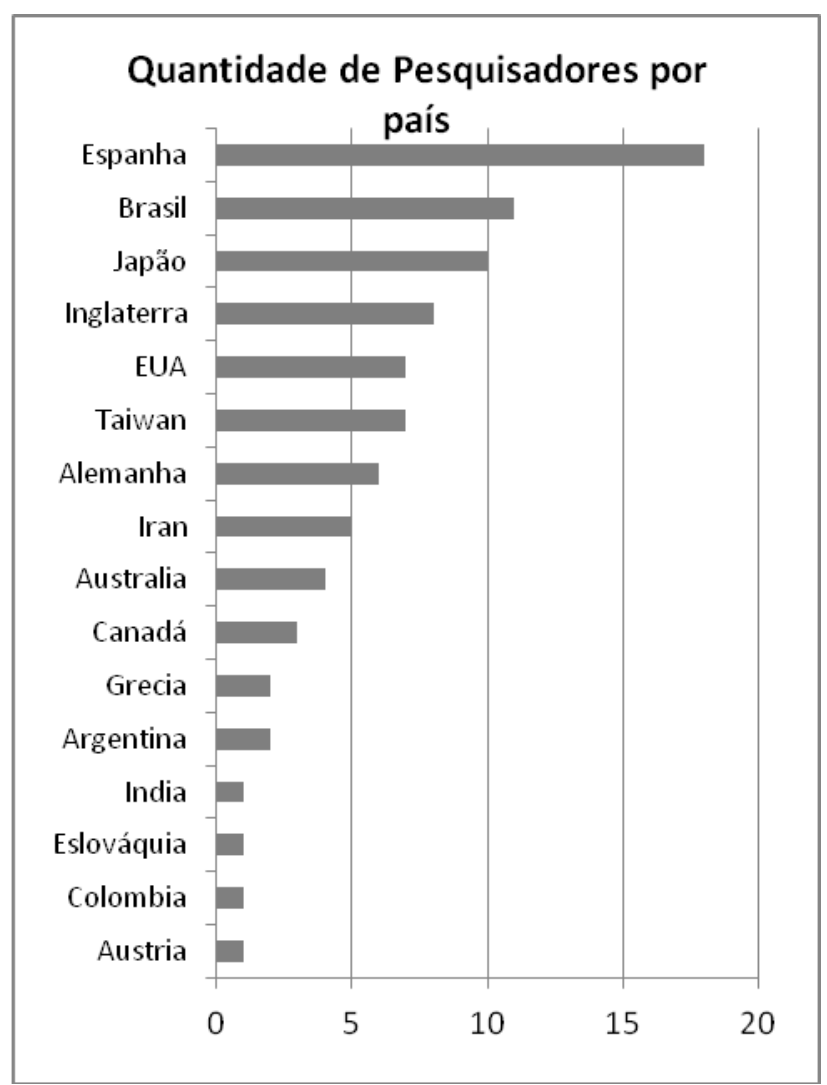

Figura 2: Quantidade de pesquisadores por país

Observou-se que os eventos GROUP (GROUP Supporting group work) e IEEE TLT (IEEE Transactions on 
Learning Technologies) lideram o ranking, ambos com $10 \%$, seguidos por ICALT (ICALT Advanced Learning Technologies), Computers in Human Behavior e WI-IAT (Web Intelligence and Intelligent Agent Technology), que apresentam aproximadamente $6,7 \%$. Os quase $84 \%$ restantes estão distribuídos entre as demais conferências e revistas científicas, conforme Figura 3.

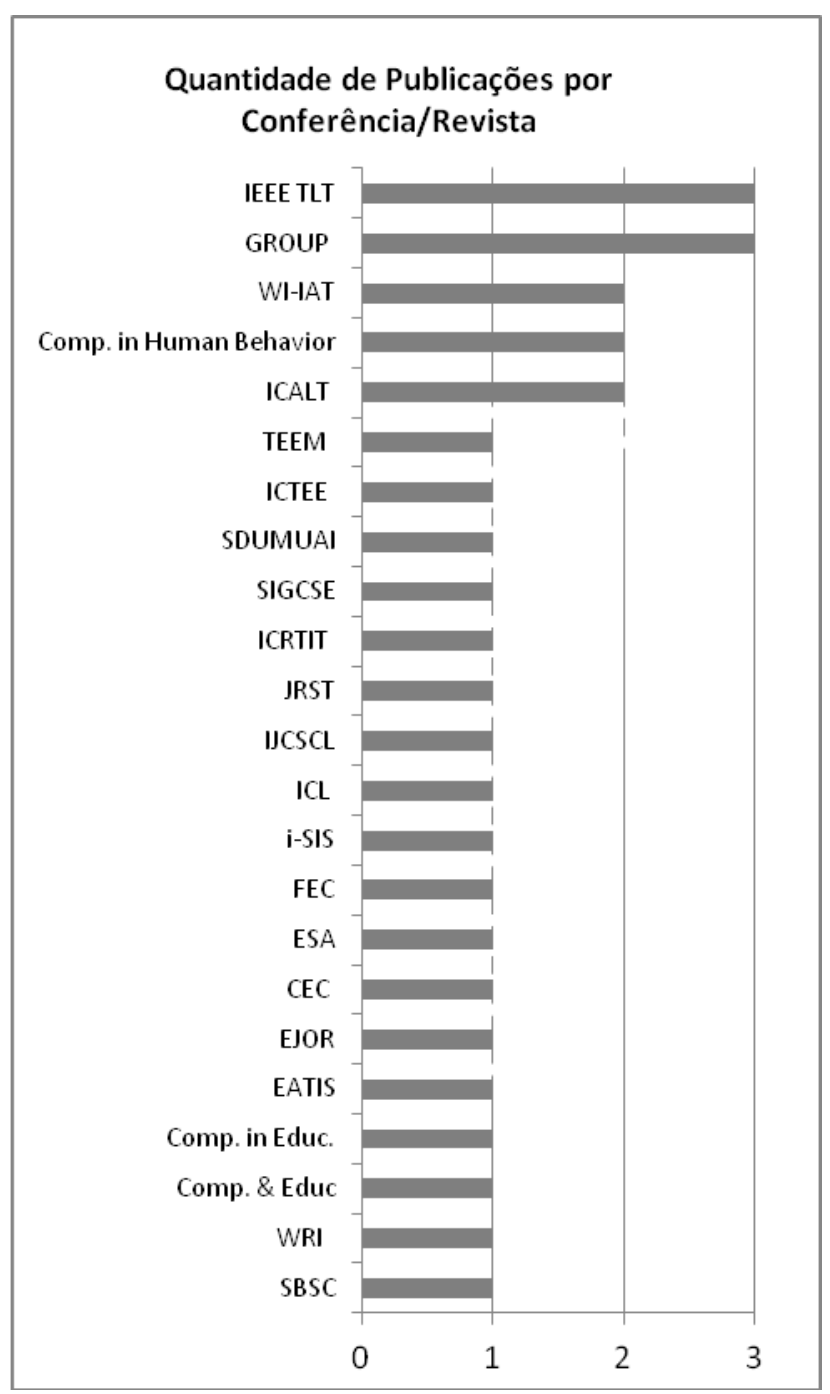

Figura 3: Quantidade de publicações por conferência/revista

É importante destacar que as publicações na área são, em grande parte, em conferências científicas. Tal fato pode ser um indicador que pesquisadores têm procurado em grande parte os eventos para suas publicações. Apesar de alguns periódicos possuírem publicação bimestral ou trimestral, eles apresentam um tempo de resposta bem maior quando comparado ao processo de escolha de artigos pelas conferências.

\subsection{Informações específicas dos trabalhos primários}

Após a compreensão das informações gerais, os trabalhos primários foram analisados a partir da leitura completa de seu conteúdo para extrair as informações específicas e, dessa forma, contribuir com pesquisas futuras. As informações específicas mapeadas estão descritas nesta seção.

\subsubsection{Abordagens para formação de grupos encontradas nos trabalhos primários}

A abordagem implementada para atribuir estudantes em grupo estabelece aspectos relevantes para o sucesso da aprendizagem colaborativa, tendo em vista as possibilidades de configuração e de combinação dos membros nos grupos.

De acordo com a leitura e análise individual dos trabalhos, foram identificadas diferentes abordagens de pesquisa em formação de grupos colaborativos. A maioria dos trabalhos, sendo eles $42 \%$, apresenta um método para formação de grupos baseado no contexto da interação dos estudantes em atividades colaborativas $[33,42,40,18$, $22,16,23,19,30,24,37,28]$. Em seguida, 34\% dos trabalhos têm foco em otimização de algoritmos para formar os grupos [21, 39, 34, 41, 26, 43, 35, 32, 27, 15]. Há também abordagens que buscam formar grupos com base no Estilo de Aprendizagem dos estudantes (11\%) [20, 31, 17]. Outras contribuições dos trabalhos primários (7\%) têm foco na implementação de sistemas baseados em Web $[25,38]$ para formar grupos efetivos, utilizando, por exemplo, interface de usuário para configuração dos agrupamentos. Há ainda trabalhos que apresentam abordagens baseadas em ontologias (3\%) [29] e outros (3\%) que focam suas pesquisas em metodologias de avaliação da formação de grupos [36]. A Figura 4 ilustra as abordagens encontradas. 


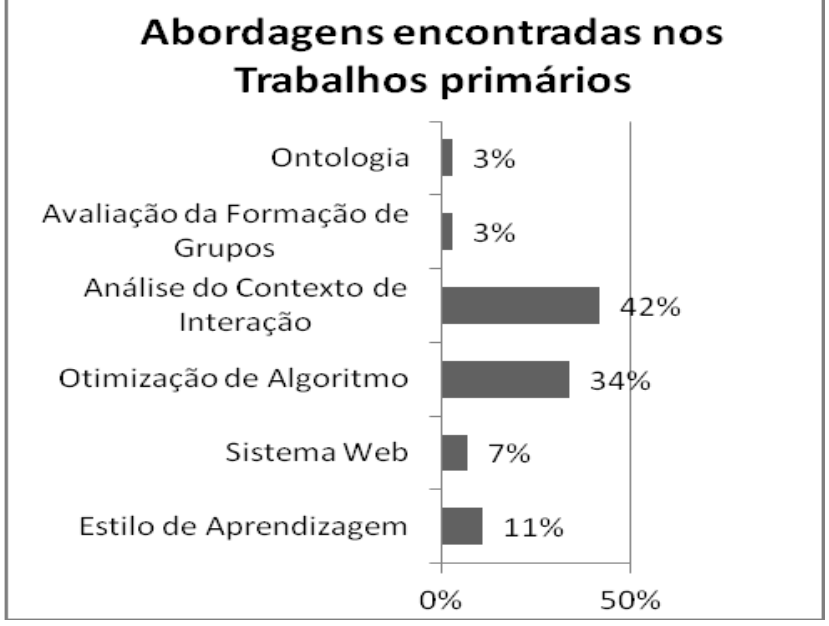

Figura 4: Abordagens encontradas nos Trabalhos primários

\subsubsection{Tipos de grupos encontrados nos Tra- balhos primários}

O tipo de grupo é um dos parâmetros utilizados na configuração de grupos, assim como o tamanho, a duração e os objetivos de aprendizagem. Diversos tipos de grupos encontrados na literatura caracterizam as informações utilizadas na hora de atribuir os membros.

Os trabalhos primários analisados apresentam diferentes tipos de grupos. De forma geral, eles propõem grupos homogêneos e/ou heterogêneos em relação a determinadas características. Verificou-se que $45 \%$ dos trabalhos propõem tipos de grupos como heterogêneos em relação aos dados do perfil do aluno, tais como a turma, a região em que se encontra, o gênero, o tipo de personalidade ou o nível de habilidade, como por exemplo a habilidade com programação $[33,21,36,34,41,43$, $35,28,16,22,24,29,37]$. Em seguida, tem-se $28 \%$ que considera o tipo de grupo heterogêneo e homogêneo por desempenho em atividade de colaboração, obtido pelo nível de interação entre os estudantes [25, 38, 42, 23, 19 , $40,30,27]$. O tipo de grupo por estilo de aprendizagem pode ser encontrado em $10 \%$ dos trabalhos analisados $[20,31,17]$. O tipo de grupos mistos, que combinam os perfis de grupos anteriores, são encontrados em $14 \%$ dos trabalhos analisados $[39,26,18,15]$. Outros trabalhos utilizam o tipo de grupo por nível de habilidade sócioafetiva obtido de questionário aplicado aos alunos (3\%) [32].

\subsubsection{Trabalhos que relacionam formação de grupos e E-learning}

A formação de grupos de aprendizagem aplicada em E-learning constitui uma promissora linha de pesquisa em aprendizagem colaborativa apoiada por computador, tendo em vista as possibilidades ainda desconhecidas pela comunidade científica. Pesquisas revelam as possibilidades de criação de novos espaços para a co-aprendizagem e de estímulo ao desenvolvimento de habilidades fundamentais para elevar o potencial de alunos para a resolução de problemas, a criatividade e a auto regulação da aprendizagem. Analisando os trabalhos primários, verificou-se, conforme Figura 5, que $69 \%$ dos trabalhos relacionam o estudo sobre Formação de Grupos e E-learning simultaneamente em suas pesquisas; enquanto que $31 \%$ dos trabalhos primários tratam apenas de Formação de Grupos, em geral, trazendo contribuições do ponto de vista da otimização de algoritmo e de modelos teóricos de agrupamento.

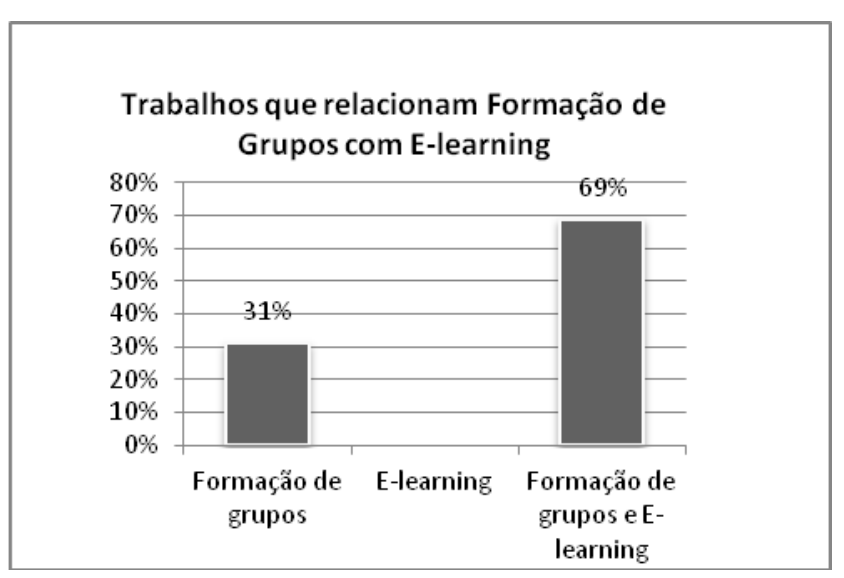

Figura 5: Estudos que relacionam formação de grupos em E-learning

\subsubsection{Ambientes Virtuais de Aprendizagem utilizados nas pesquisas dos trabalhos primários}

Diversos trabalhos primários aqui apresentados utilizam em seus estudos Ambientes Virtuais de Aprendizagem - AVA, caracterizados por serem sistemas de aprendizagem online, para obter informações dos estudantes, para formar grupos automaticamente ou para colocar os estudantes em atividades que promovam a colaboração.

A análise dos trabalhos primários permitiu identificar 10 (dez) ambientes de apoio à aprendizagem colaborativa. Verificou-se que, embora $27,5 \%$ não especificarem e $13,8 \%$ não utilizarem algum ambiente AVA em suas 
pesquisas, $58,7 \%$ dos trabalhos analisados relataram a utilização de pelo menos um ambiente virtual de aprendizagem, compreendendo os sistemas: MOODLE $(10,3 \%)$, COLLECE $(6,9 \%)$, TANGOW $(3,4 \%)$, BSCW $(3,4 \%)$, PQA-C $(3,4 \%)$, Rede Social $(3,4 \%)$, PopCorm $(3,4 \%)$, eXpresser (3,4\%), Ambiente L (3,4\%) e WebClassRAPSODY $(3,4 \%)$, conforme Figura 6.

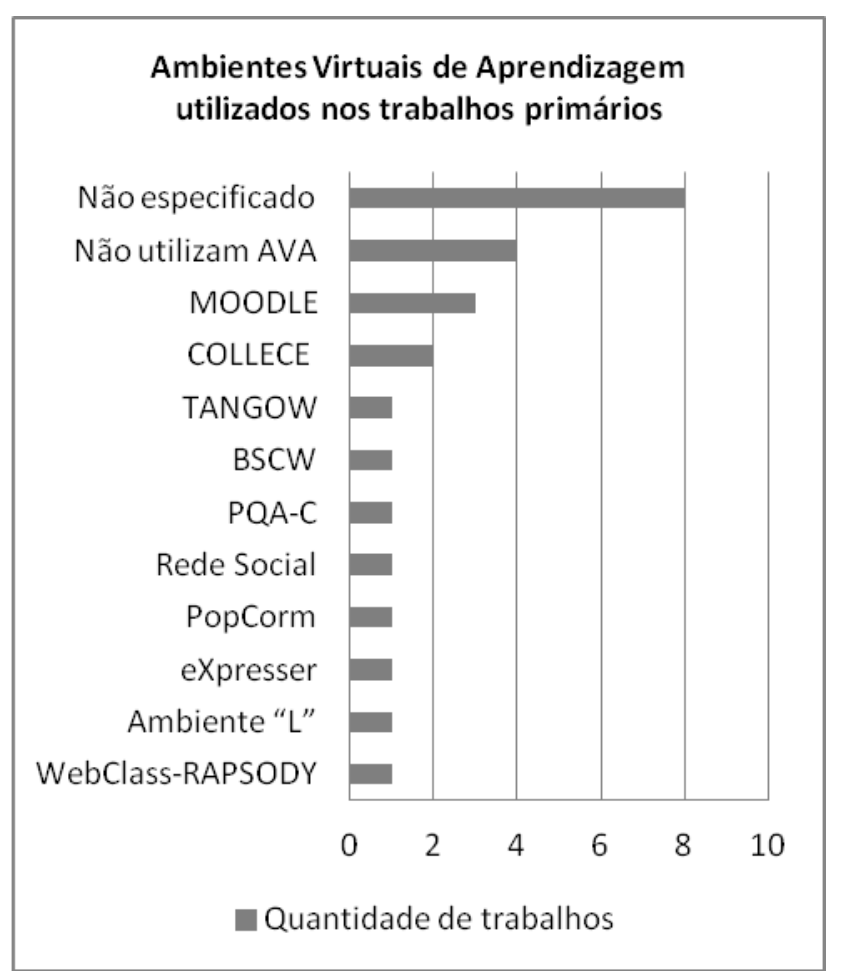

Figura 6: Ambientes virtuais de aprendizagem utilizados nas pesquisas dos trabalhos primários

\subsubsection{Trabalhos primários que analisam a interação dos estudantes em atividades online}

Extrair informações dos estudantes a partir do comportamento deles no trabalho colaborativo em sistemas $E$ learning pode ser uma estratégia eficaz para formar grupos eficientes em atividades online. Esses sistemas permitem o diálogo e a ação através das ferramentas de comunicação e de interação para apoiar o trabalho em grupo, tais como fóruns, chats, blogs, wikis, áudio conferência, vídeo conferência, quadro branco compartilhado, ferramenta de brainstorming, entre outras. Esta análise pretende avaliar os trabalhos primários com abordagens que consideram as interações dos estudantes em ambientes de aprendizagem online.

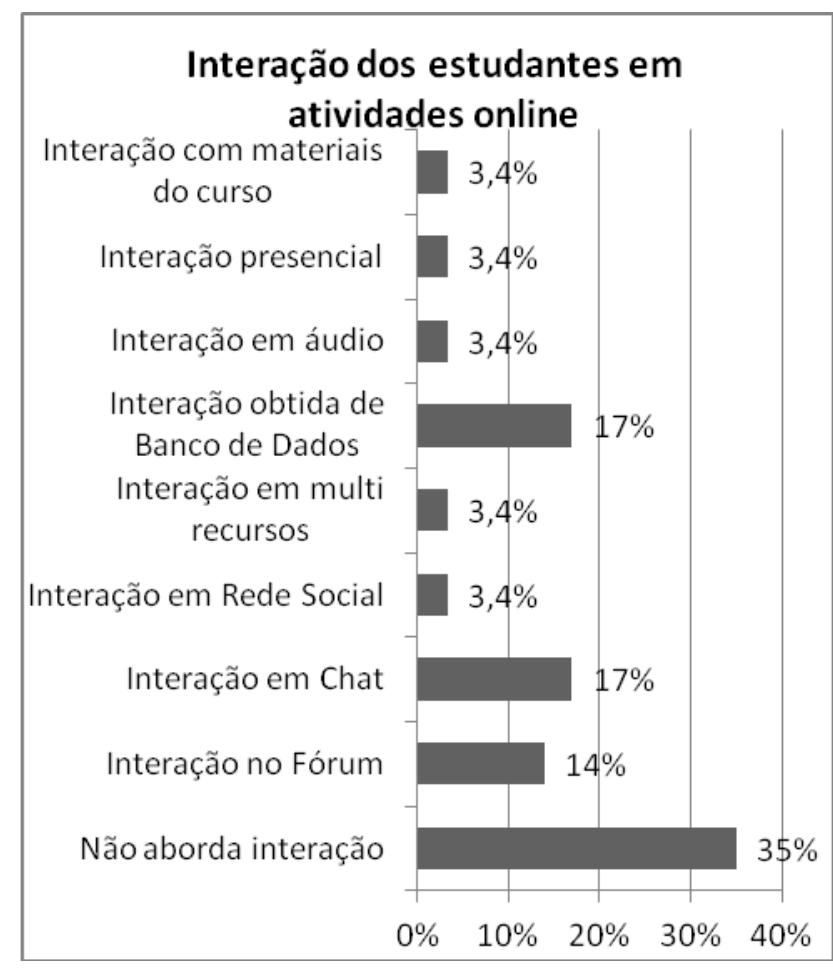

Figura 7: Abordagens que analisam a interação dos estudantes

Verificou-se, conforme Figura 7, que embora 35\% dos trabalhos não analisarem a interação dos estudantes, a maioria $(65 \%)$ dos trabalhos primários consideram a interação entre os grupos por meio de diferentes perspectivas. A interação dos estudantes em atividade colaborativa por meio do recurso de chat, por exemplo, é analisada em $17 \%$ dos trabalhos considerados primários. Em mesma quantidade, os dados referentes à colaboração armazenados em banco de dados de ambientes de aprendizagem online são analisados em $17 \%$ dos trabalhos; enquanto que a interação no recurso de Fórum para a composição dos grupos é analisada em 14\%. Já outros trabalhos analisam a interação dos estudantes em atividades de colaboração utilizando dados de comportamento em Rede Social (3,5\%), dados registrados em áudio $(3,4 \%)$ e dados da interação com materiais do curso. Há ainda 3,4\% dos trabalhos que consideram a interação de múltiplos recursos de comunicação e outros $3,4 \%$ que consideram a interação em atividades colaborativas na modalidade presencial.

\subsubsection{Algoritmos utilizados nos estudos dos trabalhos primários para formação de grupos}

Existem diferentes estratégias para a formação de grupos. Em geral, a formação de grupos de estudantes 
pode ser feita de forma manual ou automática, sendo esta no próprio ambiente do sistema de E-learning, quando este incorporar tal recurso, ou utilizando algum algoritmo de agrupamento em softwares próprios para o seu uso.

A partir da análise dos trabalhos primários, verificouse que $52 \%$ dos trabalhos não mencionaram o uso de algum algoritmo ou, no caso do uso de sistema que permite o agrupamento automático, não especificaram o seu funcionamento. Nos demais, o algoritmo Genético e o algoritmo PSO (Particle Swarm Optimization) foram utilizados nas pesquisas de $10,3 \%$ dos trabalhos primários cada. Observou-se ainda trabalhos que utilizaram outros algoritmos, a saber: A priori $(3,4 \%)$; Força bruta $(3,4 \%)$; K-NN (3,4\%); IGLS (3,4\%); Random Walk (3,4\%); Busca em largura $(3,4 \%)$; Tabu search $(3,4 \%)$, e; k-means $(3,4 \%)$, conforme Figura 8 .

\begin{tabular}{|r|r|}
\hline \multicolumn{2}{|c|}{ Algoritmos utilizados nos trabalhos } \\
primários \\
Não especificado & $10,3 \%$ \\
Particle Swarm... & 10,3\% \\
Algoritmo Genético & $3,4 \%$ \\
K-means & $3,4 \%$ \\
Tabu search & $3,4 \%$ \\
Busca em largura & $3,4 \%$ \\
Random Walk & $3,4 \%$ \\
IGLS & $3,4 \%$ \\
k-NN & $3,4 \%$ \\
Força bruta & $3,4 \%$ \\
A priori & 3 \\
\hline
\end{tabular}

Figura 8: Algoritmos utilizados nos trabalhos primários

\subsubsection{Responsável pela formação dos grupos segundo os trabalhos primários}

Criar grupos efetivos para o trabalho colaborativo requer definir o responsável pela seleção dos membros. Há sugestões que afirmam que formar os grupos seja de responsabilidade do professor, tendo em vista sua grande carga de conhecimento acerca dos pontos fortes e fracos dos alunos.

A análise dos trabalhos permitiu verificar que, embora a maioria $(44,8 \%)$ não tenham especificado quem de fato é o responsável por criar os grupos, $38 \%$ afirmam ser de responsabilidade do professor/ instrutor a tarefa de sele- cionar os membros; enquanto que $10,4 \%$ consideram o uso de algoritmos para substituir o papel dos professores em atribuir os estudantes aos grupos. No entanto, 3,4\% dos trabalhos considera o envolvimento dos tutores com os alunos, o que lhes confere a tarefa de selecionar os alunos a partir dos critérios e preferências definidos. Outros 3,4\% dos trabalhos primários consideram em seus estudos a atribuição dos estudantes em grupo ser autoselecionada, ou seja, os próprios alunos é quem devem ser os responsáveis por criar grupos, conforme Figura 9.

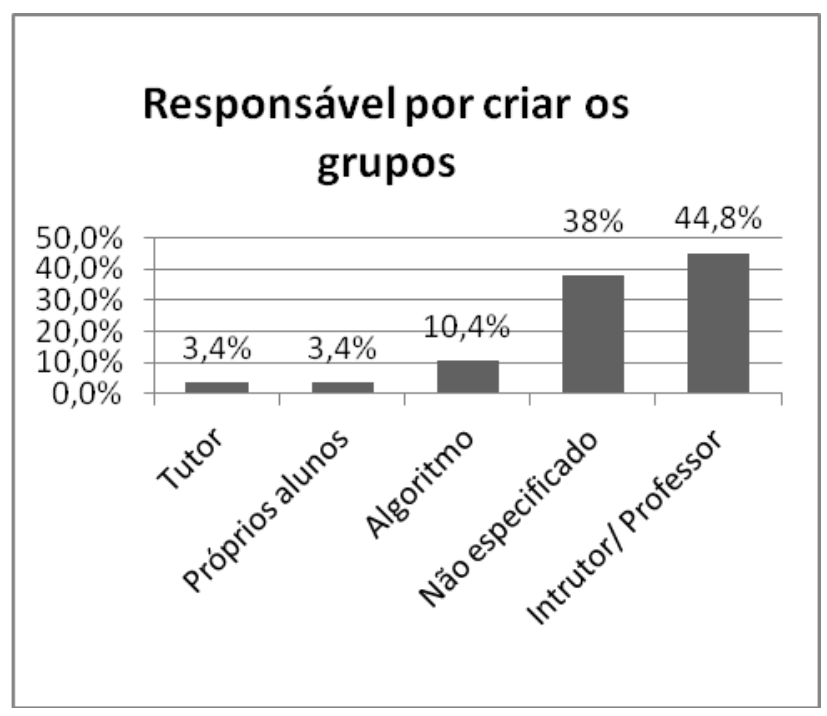

Figura 9: Responsável por criar grupos segundo os trabalhos primários

\subsubsection{Propostas de estudos futuros encontra- das nos trabalhos primários}

Esta seção se dedica a apresentar as possibilidades de pesquisa sobre formação de grupos em E-learning, conforme apresentadas nos trabalhos primários analisados. Foram consideradas as possibilidades de estudo futuro as linhas de pesquisas ainda não explorados e/ou problemas a serem ainda solucionados.

Os trabalhos primários analisados apresentam diversos temas de propostas para estudos futuros. Os autores relatam, por exemplo, a construção de interface para usuários de sistemas que possibilitam a criação de grupos, a realização de experimentos reais em ambientes virtuais de aprendizagem, a realização de testes considerando novas características dos estudantes e novos critérios de configuração para composição de grupos, entre outras temáticas.

Os temas de pesquisas foram categorizados e dispostos na Tabela 2 . 


\begin{tabular}{l|l}
\hline \multicolumn{1}{c|}{ Categorização de pesquisas futuras } & \multicolumn{1}{c}{ Percentual } \\
\hline Analisar os fatores cognitivos da colaboração & $3,4 \%$ \\
\hline Incluir o apoio do tutor na colaboração & $3,4 \%$ \\
\hline Desenvolver agente assistente & $7 \%$ \\
\hline Implementar interface de software & $7 \%$ \\
\hline Desenvolver algoritmos adicionais & $20 \%$ \\
\hline Aplicação em ambientes e domínios diferentes & $20 \%$ \\
\hline $\begin{array}{l}\text { Melhorar a definição/ incluir novos critérios de } \\
\text { agrupamento }\end{array}$ & $31 \%$ \\
\hline Novos experimentos com dados reais & $45 \%$
\end{tabular}

Tabela 2: Pesquisas futuras encontradas nos trabalhos primários

Verificou-se que a maioria, representando $45 \%$ dos trabalhos primários, propõe realizar novos experimentos incluindo dados reais. Observou-se ainda que melhorias na definição dos atributos e a inclusão de novos critérios de agrupamento são temas de pesquisas futuras encontradas em $31 \%$ dos trabalhos primários. Outros temas em destaque que foram encontrados em $20 \%$ dos trabalhos são a aplicação da abordagem em ambientes e domínios diferentes e a melhoria e desenvolvimento de algoritmos adicionais; enquanto que a implementação de uma interface de usuário do sistema de agrupamento é um tema proposto em cerca de 7\% dos trabalhos analisados. Outros $7 \%$ dos trabalhos propõe desenvolver um agente assistente para monitorar a colaboração no ambiente virtual. As pesquisas futuras que pretendem analisar os fatores cognitivos da colaboração e a inclusão do apoio do tutor na mediação e colaboração no ambiente são temas encontrados em 3,4\% dos trabalhos.

\subsection{Tipos de Grupos}

Com base nos resultados do mapeamento sistemático, verifica-se que há uma ampla diversidade de formas de agrupamento a ser explorada. Diversas abordagens encontradas na literatura, em geral, propõem estratégias para formação de grupos homogêneos [23, 19], heterogêneos [43] e mistos [39, 26, 18, 15], utilizando diferentes critérios. Diante disso, este trabalho introduz um novo tipo de agrupamento, chamado de grupos complementares, que permite a complementaridade das características em grupos de estudantes.

\subsubsection{Representação da distância}

A distância $d$ entre dois estudantes $x$ e $y$ pode ser definida como a diferença entre suas características, conforme Equação 1, onde $i$ representa uma dada característica $C$ para $x$ e $y$. $d\left(x_{i}, y_{i}\right)=\left|C x_{i}-C y_{i}\right|$

\subsubsection{Grupos homogêneos}

Para obtenção de grupos homogêneos, o método heurístico busca minimizar a diferença entre pares de objetos dentro de um conjunto de dados. Pode ser definido como sendo a diferença entre os pares de estudantes $x$ e $y$ dentre todas as características $C$, conforme Equação 2 com $i$ variando entre 1 e $q$ características.

$$
\min d\left(x_{i}, y_{i}\right)=\sum_{1 \leq i \leq q}\left|C x_{i}-C y_{i}\right|
$$

\subsubsection{Grupos heterogêneos}

Grupos heterogêneos são grupos formados por estudantes o mais distintos possível entre si, em relação a um ou mais critérios de agrupamento. Um grupo por habilidades heterogêneas pode ser obtido buscando maximizar a distância entre as habilidades dos estudantes. Ao contrário do grupo homogêneo, a soma das diferenças entre os pares de estudantes $x$ e $y$ das características $C$ é maximizada, conforme Equação 3 com $i$ variando entre 1 e $q$ características.

$$
\max d\left(x_{i}, y_{i}\right)=\sum_{1 \leq i \leq q}\left|C x_{i}-C y_{i}\right|
$$

\subsubsection{Grupos complementares}

Grupo complementar pode ser definido como o agrupamento cujos membros se complementam em relação a habilidades distintas. Os estudantes deverão ser atribuídos ao grupo de forma que um apresente em uma dada habilidade um nível maior que o outro e que este apresente em uma dada habilidade distinta um nível maior que aquele.

Em termos matemáticos, um par de estudantes é considerado complementar quando a soma das diferenças entre $x$ e $y$ nas habilidades $i$ e $j$, considerando $x>y$ em $i$ e $x<y$ em $j$, com $i \neq j$, é maximizada, conforme descrito nas Equações 4 e 5 .

$$
\begin{aligned}
& \max d(x, y)=\left|C x_{i}-C y_{i}\right|+\left|C x_{j}-C y_{j}\right| \\
& \max \left(x_{i}, y_{i}\right)=\left\{\begin{array}{l}
x_{i}, s e \max \left(x_{j}, y_{j}\right)=y_{j} \\
y_{i}, s e \max \left(x_{j}, y_{j}\right)=x_{j}
\end{array}\right.
\end{aligned}
$$

O procedimento utilizado na implementação da modelagem matemática, para obtenção de grupos complementares, utiliza a estratégia de calcular a distância entre dois objetos considerando a maior distância entre um par de exemplos, de maneira a garantir a complementaridade descrita na Equação 5. Após verificar se o par é comple- 
mentar, o algoritmo aloca os objetos a um grupo disponível. Para saber se o grupo está disponível, considera-se o tamanho estimado para cada grupo. Em [28] é utilizado experimentos com de grupos de três e quatro membros. Para o experimento descrito na Seção 4.3.5 os dados de estudantes foram atribuídos a grupos de tamanho quatro e cinco.

\subsubsection{Experimento com Grupos complemen- tares}

O experimento para formação de grupos complementares considerou os dados dos níveis de conhecimento de estudantes em relação às habilidades de Linguagem de Programação C - LPC e de Estrutura de Controle - EC, extraídos de banco de dados de sistema acadêmico de um curso superior a distância na área da ciência da computação. Essas habilidades estão representadas respectivamente pelas habilidades LPC e EC na Tabela 3, que foram desenvolvidas na disciplina de Introdução à Programação do referido curso.

\begin{tabular}{|c|c|c|}
\hline \multirow[t]{2}{*}{ Estudante } & \multicolumn{2}{|c|}{ Habilidade } \\
\hline & LPC & EC \\
\hline 01: & 5,8 & 6,7 \\
\hline 02: & 6,2 & 4,9 \\
\hline 03: & 6,7 & 7,8 \\
\hline 04: & 5 & 2,4 \\
\hline 05: & 4,3 & 1 \\
\hline 06: & 6,3 & 8,3 \\
\hline 07: & 6,8 & 8,2 \\
\hline 08: & 2,7 & 3,2 \\
\hline 09: & 5,5 & 2,9 \\
\hline 10: & 5,3 & 5 \\
\hline 11: & 4,7 & 1,5 \\
\hline 12: & 3,3 & 2,2 \\
\hline 13: & 4,9 & 1,9 \\
\hline 14: & 5,4 & 1,6 \\
\hline 15: & 5,6 & 7,7 \\
\hline 16: & 5,1 & 2 \\
\hline 17: & 5,8 & 4,2 \\
\hline 18: & 5,9 & 6,4 \\
\hline 19: & 6,3 & 5,4 \\
\hline 20: & 2,5 & 2,1 \\
\hline
\end{tabular}

\begin{tabular}{l|l|l} 
21: & 5,7 & 7,6 \\
\hline $22:$ & 6 & 4,1 \\
\hline $23:$ & 2,2 & 7,4 \\
\hline $24:$ & 5,2 & 4,6 \\
\hline $25:$ & 4,8 & 7,9 \\
\hline
\end{tabular}

Preferência por tamanho do grupo:4

Tamanho alternativo para os grupos:5

Tabela 3: Dados de entrada para obtenção de grupos complementares

O experimento utilizou o procedimento descrito na Seção 4.3.4, implementando a medida de distância e a propriedade apresentadas nas Equações 4 e 5 . Utilizou-se na linguagem Java para a implementação dos procedimentos e a instanciação da medida de distância para obtenção de grupos complementares. A Tabela 4 apresenta os resultados da execução com 6 grupos criados a partir do total de 25 objetos.

\begin{tabular}{|c|c|c|c|c|c|c|}
\hline \multicolumn{7}{|c|}{ Grupos complementares por habilidade em programação } \\
\hline & $\begin{array}{l}\text { Grupo } \\
1\end{array}$ & $\begin{array}{l}\text { Grupo } \\
2\end{array}$ & $\begin{array}{l}\text { Grupo } \\
3\end{array}$ & $\begin{array}{l}\text { Grupo } \\
4\end{array}$ & $\begin{array}{l}\text { Grupo } \\
5\end{array}$ & $\begin{array}{l}\text { Grupo } \\
6\end{array}$ \\
\hline & $\begin{array}{l}14 \\
23 \\
16 \\
25\end{array}$ & $\begin{array}{l}15 \\
22 \\
05 \\
08\end{array}$ & $\begin{array}{l}17 \\
21 \\
11 \\
20\end{array}$ & $\begin{array}{l}09 \\
10 \\
01 \\
02\end{array}$ & $\begin{array}{l}12 \\
13 \\
18 \quad 19\end{array}$ & $\begin{array}{l}03 \\
06 \\
24 \\
0407\end{array}$ \\
\hline 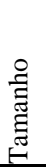 & 4 & 4 & 4 & 4 & 4 & 5 \\
\hline
\end{tabular}

Total de dados: 25; Total de grupos alocados: 6 .

Tabela 4: Agrupamentos complementares em relação aos níveis de conhecimento nas habilidades Linguagem de programação $\mathrm{C}$ e Estrutura de controle

Verificou-se que o método heurístico implementado foi eficiente por formar grupos de estudantes o mais complementar possível em relação às habilidades em Programação. Os pares de estudantes mais complementares foram atribuídos aos primeiros grupos com tamanhos de até quatro membros. O Grupo 1 como sendo o mais complementar possível, seguido dos Grupos 2, 3, 4 e 5 alocados com tamanho de quatro membros e o Grupo 6 com tamanho flexibilizado para um tamanho aproximado ao desejado, conforme sugerido em [44]. 


\section{Conclusões}

A procura por estratégias de ensino e de aprendizagem que promovam e fortaleçam o diálogo e a colaboração, sobretudo na educação a distância, tem sido tema de crescente destaque na literatura científica nas últimas décadas, tendo em vista os diversos modos de interação possibilitados pelos ambientes de aprendizagem $E$ learning. Recentemente pesquisas têm investigado abordagens de formação de grupos para a aprendizagem colaborativa a distância, o que tem permitido à comunidade científico-acadêmica experimentar as diferentes maneiras de atribuir estudantes em grupos e as melhores práticas de aprendizagem colaborativa com o suporte computacional.

Identificar as características mais decisivas sobre os estudantes, definir critérios claros e objetivos e pensar situações de aprendizagem colaborativa pode ser um caminho para formar grupos efetivos do ponto de vista pedagógico e capaz de elevar o desempenho do aprendizado dos alunos através do trabalho em grupo.

O mapeamento sistemático teve por objetivo fornecer uma visão geral das abordagens e das estratégias sobre formação de grupos em E-learning e contribuir com temas de estudos futuros nessa área. As etapas do mapeamento apresentadas permitiram encontrar 29 (vinte e nove) trabalhos primários, entendidos como artigos científicos originais publicados em conferências e revistas relevantes na área. Essa quantidade deve-se ao fato de ser uma sub-área bastante promissora e relativamente recente dentro da área de formação de grupos de aprendizagem, conforme aumento gradativo das publicações nos últimos anos, o qual foi ilustrado na Figura 1.

Os resultados deste mapeamento sistemático evidenciam que diversas abordagens para formação de grupos podem contribuir para a aprendizagem colaborativa em E-Learning, haja vista as múltiplas formas de interação e as variadas combinações de características dos alunos encontradas a partir da análise do contexto das interações. Dado, também, o fato de que a grande maioria dos estudos se preocupam em analisar as interações de estudantes por meio de pelo menos um recurso de comunicação presente em ambientes virtuais de aprendizagem.

Evidencia-se ainda que as publicações na área tem se dedicado a mineração de dados educacionais para extrair informações peculiares do comportamento dos alunos em colaboração. E que as possibilidades de tipos de grupos associadas ao uso de algoritmo de agrupamento podem auxiliar professores na tarefa de seleção dos alunos e de otimização de grupos, especialmente em E-learning.

A abordagem definida para formação de grupos complementares possibilitou a diversificação dos tipos de grupos discutidos pela comunidade científico-acadêmica, viabilizando uma maior abrangência das preferências e dos critérios para a formação de grupos de aprendizagem.

Os tipos de grupos descritos neste estudo apresentamse como uma ferramenta para apoiar o professor no processo de criação de grupos de estudantes. O experimento para obtenção de grupos complementares, em específico, consistiu em sugestão para dar suporte a novos estudos com dados educacionais.

A formação de grupos, baseada na obtenção das informações de estudantes por meio da mineração de dados educacionais, cuja imensidão de informações é, muitas vezes, desconhecida por seus usuários, pode ser uma alternativa para viabilizar e identificar as competências fundamentais para o trabalho em grupo.

Como trabalhos futuros, pretende-se explorar diferentes critérios e perfis de grupos para apoiar a tarefa de formação de grupos colaborativos, realizar experimentos no contexto da educação a distância e investigar o impacto dessa abordagem sobre o desempenho de estudantes.

\section{Referências}

[1] J. A. Brito, I. J. Melo Filho, R. S. Carvalho, R. M. Melo, A. S. Gomes. Interfaces colaborativas para atividades assíncronas em fórum de discussão. In Anais do XXII Simpósio Brasileiro de Informática na Educação - XVII WIE, Aracajú, Sergipe, Brasil, páginas 876-879, 2011.

[2] K. J. Chapman, M. Meuter, D. Toy, L. Wright, Can't We Pick Our Own Groups? The Influence of Group Selection Method on Group Dynamics and Outcomes. Management Education, 30(4): 557-569, 2006.

[3] P. Dillenbourg. What do you mean by collaborative learning? In P. Dillenbourg (ed.), Collaborative learning: Cognitive and computational approaches, páginas 1-16, Elsevier Science, Amsterdam, Holanda. 1999.

[4] R. Hubscher, Assigning students to groups using general and context specific criteria. IEEE Transactions on Learning Technologies, 3(3): 178-189, 2010.

[5] A. Inaba, T. Supnithi, M. Ikeda, R. Mizoguchi, J. Toyoda, How Can We Form Effective Collaborative Learning Groups?. In Proceedings of the 5th International Conference on Intelligent Tutoring Systems, Montreal, Canadá, páginas 282291, 2000. 
[6] M. B. Josué Neto, A. S. Gomes, I. J. S. Aquino Júnior. Representação das Atividades Sociais em Ambientes CSCL Utilizando Mecanismos de Percepção. In Anais do XVI Simpósio Brasileiro de Informática na Educação - SBIE - Juiz de Fora, Minas Gerais, Brasil, páginas 265-275, 2005.

[7] S. Mahenthiran, P. J. Rouse, The Impact of Group Selection on Student Performance and Satisfaction. Educational Management, 14(6): 255-265, 2000.

[8] M. Muehlenbrock, Learning Group Formation Based on Learner Profile and Context, International Journal on E-Learning, 5(1):19-24, 2006.

[9] A. S. Palincsar, A. L. Brown, Reciprocal Teaching of Comprehension-Fostering and Comprehension-Monitoring Activities. Cognition and Instruction, 1( 2):117-175, 1984.

[10] K. Petersen, R. Feldt, S. Mujtaba, M. Mattsson. Systematic mapping studies in software engineering. In Anais do XII International Conference on Evaluation and Assessment in Software Engineering, páginas 68-77, 2008.

[11] J. Roschelle, S. Teasley. The construction of shared knowledge in collaborative problem solving. In O'Malley, C.E., (ed.), Computer Supported Collaborative Learning, páginas 69-97, Spring-Verlag, Heidelberg, Alemanha. 1995.

[12] B. Rosenshine, C. Meister, Reciprocal Teaching: A Review of the Research. Rev. of Educational Research, 64(4): 479-530, 1994.

[13] P. D. Rumrill, S. M. Fitzgerald, Speaking of research: Using narrative literature reviews to build a scientific knowledge base. Work: A Journal of Prevention, Assessment, and Rehabilitation, 16(2): 165-170, 2001.

[14] E. L. Tecchio, T. S. Nunes, S. M. Moretto, M. B. L. Dalmau, P. A. Melo, Competências fundamentais ao tutor de ensino à distância.Colabor@ - Revista Digital da CVA - Ricesu, 6 (21):1-12, 2009.

[15] S. Abnar, F. Orooji, F. Taghiyareh, An Evolutionary Algorithm for Forming Mixed Groups of Learners in Web Based Collaborative Learning Environments. In Proceedings of the IEEE International Conference Technology Enhanced Education (ICTEE), Amritapuri, Kerala, Índia, páginas 1-6, 2012.
[16] W. Alandeom, T. Oliveira, D. Sadler, Interactive Patterns and Conceptual Convergence During Student Collaborations in Science. Journal of Research in Science Teaching, 45(5): 634-658, 2008.

[17] E. Alfonseca, R. M. Carro, E. Martín, A. Ortigosa, P. Paredes, The impact of learning styles on student grouping for collaborative learning: a case study. User Model User-Adap Inter, 16:377401, 2006.

[18] S. Brauer, T. C. Schmidt, S. Brauer, T. C. Schmidt, Group Formation in e Learning-enabled Online Social Networks. In Proceedings of the IEEE Congress on Evolutionary Computation (CEC 2015), Sendai, Japão, 3296-3304, 2015.

[19] M. Cocea, G. D. Magoulas, User behaviourdriven group formation through case-based reasoning and clustering. Expert Systems with Applications, 39: 8756-8768, 2012.

[20] R. Costaguta, M. L. A. Menini, An Assistant Agent for Group Formation in CSCL based on Student Learning Styles. In Proceedings of the 7th Euro American Conference on Telematics and Information Systems (EATIS '14), Valparaiso, Chile, páginas 1-24, 2014.

[21] M. Craig, D. Horton, F. Pitt, Forming Reasonably Optimal Groups (FROG). In Proceedings of the 16th ACM International Conference on Supporting Group Work, Sanibel, Flórida, USA, páginas 141-150, 2010.

[22] T. Daradoumis, M. Guitert, F. Giménez, J.M. Marquès, T. Lloret, Supporting the Composition of Effective Virtual Groups for Collaborative Learning. In Proceedings of the International Conference on Computers in Education (ICCE'02), Palmerston, Auckland, Nova Zelândia, páginas 332-336, 2002.

[23] R. Duque, D. G. Pérez, A. N. Reyes, Crescencio Bravo Analyzing collaboration and interaction in learning environments. Computers in Human Behavior, 47: 42-49, 2015.

[24] E. S. J. Faria, J. M. Adán-Coello, K. Yamanaka, Forming Groups for Collaborative Learning in Introductory Computer Programming Courses Based on Students' Programming Styles: An Empirical Study. In Proceedings of the 36th Frontiers in Education Conference, San Diego, Califórnia, EUA, páginas 6-11, 2006. 
[25] T. R. Henry, Creating Effective Student Groups: An Introduction to groupformation.org. In Proceeding of the 44th ACM technical symposium on Computer science education, Denver, Colorado, USA, páginas 645-650, 2013.

[26] Y. S. Hih Lin, Y.C. Chang, C.P. Chu, Novel Approach to Facilitating Tradeoff MultiObjective Grouping Optimization. IEEE Transactions on Learning Technologies, PP(99): 1-14, 2015.

[27] T.-F. Ho, S. J. Shyu, F.-H. Wang, C.T.-J.Li, Composing High-heterogeneous and Highinteraction Groups in Collaborative Learning with Particle Swarm Optimization. In Proceedings of the World Congress on Computer Science and Information Engineering, (CSIE '09), Wilshire Grand Los Angeles, Los Angeles, USA, páginas 607-611, 2009.

[28] R. Hubscher, Assigning Students to Groups Using General and Context-Specific Criteria. IEEE Transactions on Learning Technologies, 3 (3): 178- 189, 2010.

[29] S. Isotani, A. Inaba, M. Ikeda, R. Mizoguchi, An ontology engineering approach to the realization of theory-driven group formation. ComputerSupported Collaborative Learning, (4): 445-478, 2009.

[30] D. Jagadish, Grouping in Collaborative ELearning Environment Based On Interaction among Students. In Proceedings of the IEEEInternational Conference on Recent Trends in Information Technology (ICRTIT 2014), Chennai, India, páginas 1-5, 2014.

[31] S. Liu, M. Joy, N. Griffiths, An Exploratory Study on Group Formation Based on Learning Styles. In Proceedings of the 13th IEEE International Conference on Advanced Learning Technologies, (ICALT), HaiDian, Beijing, China, páginas 95-99, 2013.

[32] J. A. B. Lopes Filho, C. C. Quarto, Clustering Algorithm for the Socio-affective Groups Formation in Aid of Computer Supported Collaborative Learning. In Anais do II Simpósio Brasileiro de Sistemas Colaborativos, Belo Horizonte, Minas Gerais, Brasil, Páginas 24-27, 2010.

[33] R. D. Medina, A. N. Reyes, D. G. Pérez, C. B. Santos, A method to form learners groups in computer-supported collaborative learning systems. In Proceedings of the First International Conference on Technological Ecosystem for Enhancing Multiculturality, Salamanca, Espanha, páginas 261- 262, 2013.

[34] J. Moreno, D. A. Ovalle, R. M. Vicari, A genetic algorithm approach for group formation in collaborative learning considering multiple student characteristics. Computers \& Education, 58: 560-569, 2012.

[35] A. Mujkanovic, D. Lowe, K. Willey, C. Guet, Unsupervised Learning Algorithm for Adaptive Group Formation: Collaborative Learning Support in Remotely Accessible Laboratories. In Proceedings of the International Conference on Information Society (i-Society 2012), London, United Kingdom, páginas 50-57, 2012.

[36] A. Ounnas, D. E. Millard, H. C. Davis, A Metrics Framework for Evaluating Group Formation. In Proceedings of the 14th ACM International Conference on Supporting Group Work, Sanibel Island, Florida, USA, páginas 221-224, 2007.

[37] Y. A. Pollalis, G.Mavrommatis, Using similarity measures for collaborating groups formation: A model for distance learning environments. European Journal of Operational Research, 193(2), 626-636, 2009.

[38] N. Rubens, M.Vilenius,T.Okamoto, Automatic Group Formation for Informal Collaborative Learning. In Proceedings of the IEEE/WIC/ACM International Joint Conferences on Web Intelligence and Intelligent Agent Technologies (WIIAT '09), Milão, Itália, páginas 231- 234, 2009.

[39] H. Sadeghi, A. A. Kardan, A novel justice-based linear model for optimal learner group formation in computer-supported collaborative learning environments. Computers in Human Behavior, 48:436- 447, 2015.

[40] I. Srba, M. Bielikova, Dynamic Group Formation As An Approach To Collaborative Learning Support. IEEE Transactions on Learning Technologies, 8 (2): 173- 186, 2015.

[41] M. R. D. Ullmann, D. J. Ferreira, C. G. CamiloJunior, S. S. Caetano, L. Assis, Formation of Learning Groups in cMoocs Using Particle Swarm Optimization. In Proceedings of the IEEE Congress on Evolutionary Computation (CEC 2015), Sendai, Japão, páginas 3296- 3304, 2015.

[42] M. Wessner, H. R. Pfister, Group Formation in Computer Supported Collaborative Learning. In Proceedings of the 2001 International ACM SIGGROUP Conference on Supporting Group Work, Boulder, Colorado, USA, páginas 24-31, 2001. 
[43] Z. Zheng, N. Pinkwart, Discrete Particle Swarm Optimization Approach to Compose Heterogeneous Learning Groups. In Proceedings of the 15th IEEE International Conference on Advanced Learning Technologies (ICALT), Atenas, Grécia, páginas 49-51, 2014.

[44] S. Zhu, D. Wang, T. Li, Data clustering with size constraints. Knowledge-Based Systems, 23(8): 883-889, 2010. 